\title{
LA METODOLOGÍA APRENDIZAJE SERVICIO COMO FACTOR EDUCATIVO DE RESILIENCIA
}

\author{
Amparo Salcedo Mateu \\ Universidad Católica de Valencia \\ salcedo.mateu@gmail.com
}

Fecha de Recepción: 26 Marzo 2019

Fecha de Admisión: 30 Abril 2019

\section{RESUMEN}

La pedagogía Aprendizaje-Servicio es una herramienta docente que une la adquisición de competencias académicas mientras presta un servicio solidario a la sociedad. La implicación de los alumnos en los proyectos, promueve una serie de valores que van a contribuir a su desarrollo integral.

La importancia de hacer partícipes a nuestros jóvenes de su educación, desde un contexto humanizado, es la mejor forma de garantizar una ciudadanía activa recubierta de sensibilidad personal y social. Desde ese paradigma, es posible construir un nuevo futuro educativo que garantice los principios éticos y morales que tanto se necesitan para mantener una sociedad digna.

Ver aumentada la capacidad de respuesta ante las adversidades académicas y emocionales, permite construir procesos resilientes. En educación, los conocimientos, las habilidades sociales y emocionales, deben estar relacionadas para que los mecanismos que derivan en resiliencia se activen.

Palabras clave: aprendizaje -servicio; resiliencia; empoderamiento; autoestima

\section{ABSTRACT}

The service learning methodology as an educational factor of resilience. The LearningService pedagogy is a teaching tool that puts together the acquisition of academic competences while providing a solidarity service to society. The involvement of students in the projects promotes a series of values that will contribute to their integral development.

The importance of involving our young people in their education, from a humanized context, is the best way to guarantee an active citizenship covered with personal and social sensitivity. From that paradigm, it is possible to build a new educational future that guarantees the ethical and moral principles that are so necessary to maintain a dignified society.

In this way, a response capacity in the face of academic and emotional adversity is increased, 


\section{LA METOdOLOGÍA APRENDIZAJE SERVICIO COMO FACTOR EDUCATIVO DE RESILIENCIA}

allowing to build resilient processes. In education, knowledge, social and emotional skills must be related so that the mechanisms that lead to resilience are activated.

Keywords: learning-service; resilience; empowerment; self esteem

\section{APRENDIZAJE-SERVICIO}

La metodología Aprendizaje-Servicio es una herramienta docente que une la adquisición de competencias académicas mientras se presta un servicio solidario a la sociedad. Su finalidad es formar a los estudiantes acercándoles a la realidad a través de proyectos socioeducativos que ayudan a fomentar el aprendizaje en valores.

Tomaremos de (Puig 2009, citado en Gil y Garzarán 2015, p.14) las características del Aprendizaje- Servicio.

Se trata de una metodología educativa utilizable en la educación formal y no formal, aplicable con diferentes poblaciones y en diferentes contextos.

Propone un servicio a la comunidad que permite el aprendizaje y la colaboración en un marco de reciprocidad entre el contexto comunitario y los servicios educativos.

Se basa en la reflexión sobre el entorno social, sobre sus dinámicas de funcionamiento y sobre los problemas y desigualdades sociales.

Requiere de la colaboración entre instituciones educativas y entidades sociales de servicio a la comunidad.

Provoca cambios en diferentes niveles, tanto individuales, grupales, institucionales o comunitarios, así como de las políticas sociales y educativas vigentes.

Requiere para su correcta implementación la implicación de los diferentes grupos de interés afectados.

Tenemos que incidir en que no todo servicio que se presta a la sociedad de una manera solidaria es Aprendizaje-Servicio, sino que hay tres condiciones indispensables que lo diferencian de cualquier otro tipo de servicio voluntario. Concretamente Tapia (2006) dice:

Que el alumno sea el protagonista de todas y cada una de las fases del proyecto, apoyado por su responsable académico.

Que adquiera los conocimientos consignados en su guía docente mientras los pone en práctica de manera solidaria.

Que todo ello esté al servicio de unas necesidades reales en la sociedad.

Estas condiciones resumen en gran medida el proyecto educativo que representa el AprendizajeServicio, dando paso a un encuentro académico-social, que va a favorecer la emergencia de cualidades emocionales resilientes.

\section{ETAPAS DE LA INVESTIGACIÓN EN RESILIENCIA}

Desde un principio los primeros estudios sobre la resiliencia se centraron en dos modelos principalmente. Modelos centrados en las variables y modelos centrados en la persona.

Los modelos centrados en las variables intentan medir el grado de adversidad y estudian las variables que aparecen en esas situaciones de adversidad. Estos modelos ofrecen información acerca de los factores de riesgo, factores protectores y la relación con las consecuencias.

Los modelos centrados en la personas, pretenden conocer cuáles son las características de las personas resilientes al enfrentarse a situaciones adversas y qué variables inciden en su formación y mantenimiento en comparación con las que no (Bermejo, 2010).

Resumiremos mediante el esquema de (Villalba, 2004 citado en Orteu 2012, p.5) las tres etapas diferenciadas en la investigación de la resiliencia. 
La primera etapa se focalizó en las fortalezas y debilidades de los individuos. El carácter, rasgo o situación de resilencialidad es que las personas poseen fortalezas selectivas y valores que les ayudan a sobrevivir en la sociedad. En la segunda etapa, la investigación quiso saber el proceso por el cual se obtienen factores de protección frente a los de vulnerabilidad. En la tercera, el interés se centra en todas aquellas situaciones en las cuales la adversidad es superada ya sean personales 0 grupales. "Siendo el paradigma común entre ellos, el que los seres vivos, tienen energía o resiliencia". (Orteu, 2012,p.5).

Tabla 2

Etapas de la investigación en resiliencia

\begin{tabular}{|c|c|c|}
\hline \multicolumn{3}{|c|}{ TRES ETAPAS EN LA INVESTIGACIÓN EN RESILIENCIA } \\
\hline & DESCRIPCIÓN & RESULTADOS \\
\hline $\begin{array}{l}\text { PRIMERA ETAPA } \\
\text { Cualidades de resiliencia }\end{array}$ & $\begin{array}{l}\text { Descripciones } \\
\text { fenomenológicas de } \\
\text { cualidades resilientes de } \\
\text { individuos y sistemas de } \\
\text { apoyo que predicen el } \\
\text { éxito personal y social }\end{array}$ & $\begin{array}{l}\text { Lista de cualidades, } \\
\text { valores o factores de } \\
\text { protección que ayudan a } \\
\text { las personas a crecer a } \\
\text { través de la adversidad } \\
\text { (autoestima, autoeficacia, } \\
\text { sistemas de apoyo...) }\end{array}$ \\
\hline $\begin{array}{l}\text { SEGUNDA ETAPA } \\
\text { El proceso y la Teoría de } \\
\text { la resiliencialidad }\end{array}$ & $\begin{array}{l}\text { Resilencialidad es el } \\
\text { proceso de poder con } \\
\text { estresores, adversidad y } \\
\text { cambio u oportunidad de } \\
\text { manera que los resultados } \\
\text { produzcan una } \\
\text { identificación, } \\
\text { fortalecimiento y } \\
\text { enriquecimiento de los } \\
\text { factores de protección. }\end{array}$ & $\begin{array}{l}\text { Describe los procesos de } \\
\text { disrupción y reintegración } \\
\text { de la adquisición de las } \\
\text { cualidades de resiliencia } \\
\text { descritas en la primera } \\
\text { etapa. Un modelo que } \\
\text { enseña a usuarios y } \\
\text { estudiantes a elegir entre } \\
\text { reintegración resiliente, } \\
\text { reintegración cómoda } \\
\text { (vuelta a la zona de } \\
\text { confort) o reintegración } \\
\text { con pérdida. }\end{array}$ \\
\hline $\begin{array}{l}\text { TERCERA ETAPA } \\
\text { Fuerzas motivacionales y } \\
\text { resiliencia innata }\end{array}$ & $\begin{array}{l}\text { Se basa en la } \\
\text { identificación } \\
\text { multidisciplinaria del } \\
\text { pensamiento postmoderno } \\
\text { y cree en las fuerzas } \\
\text { motivacionales dentro de } \\
\text { los individuos y grupos y } \\
\text { la creación de experiencias } \\
\text { que adoptan la activación } \\
\text { y utilización de esas } \\
\text { fuerzas. }\end{array}$ & $\begin{array}{l}\text { Ayuda a los clientes y } \\
\text { estudiantes a descubrir y } \\
\text { aplicar la fuerza que } \\
\text { conduce a las personas } \\
\text { hacia la auto-actualización } \\
\text { y hacia la reintegración } \\
\text { resiliente de las } \\
\text { dificultades y obstáculos } \\
\text { de la vida. }\end{array}$ \\
\hline
\end{tabular}

Fuente (Villalba, 2004; citado en Orteu, 2012,p.5) 


\section{LA METOdOLOGÍA APRENDIZAJE SERVICIO COMO FACTOR EDUCATIVO DE RESILIENCIA}

Siguiendo a Mancieaux (2003) en la actualidad la psicología positiva pone interés en la felicidad, en el modo en el que las personas refieren variables de bienestar subjetivo como la satisfacción, la esperanza y el optimismo.

En lo individual, se acentúan los rasgos positivos: capacidad de amar y de comprometerse, valentía, competencias interpersonales, sensibilidad estética, perseverancia, perdón, originalidad, espiritualidad, sabiduría.

En cuanto al grupo, se recuperan las virtudes cívicas y las instituciones que hacen evolucionar al individuo hacia una responsabilidad mejor como ciudadano, hacia la educación, el altruismo, la civilidad, la moderación la tolerancia y la ética profesional. Desarrollar a cualquier edad la autoestima, la conciencia de las posibilidades propias, el sentido de la responsabilidad, la reparación de los daños y el sufrimiento causado o sufrido no puede por menos de preparar un terreno favorable al surgimiento de la resiliencia (p.20).

\section{LA RESILIENCIA: UN CONCEPTO MULTIDIMENSIONAL}

Desde que el concepto de Resiliencia aparece en Ciencias Sociales y en Educación, han sido muchas las definiciones que se han utilizado para dar respuesta al hecho de transcender una situación que conlleva riesgo.

El termino resiliencia ha sido definido por numerosos autores pertenecientes a diversas ciencias. Becoña (2006) considera que a pesar de las diferencias utilizadas para el término hay un acuerdo al respecto dado que en general asumimos que implica competencia en respuesta al riesgo $0 \mathrm{a}$ la adversidad. En este sentido Guajardo, Ríos, y Castro (2012) afirman que:

Todas las definiciones reconocen que es una capacidad humana, más o menos universal. En todas las definiciones está presente la idea de estrés, adversidad o contexto negativo. Se comparte el enfrentamiento de aquellas adversidades por parte del sujeto y se obtiene como resultado una adaptación positiva. (p.94.).

Para hacer frente a cualquier situación que nos proporcione la oportunidad de crecer como seres humanos responsables, Casullo (2005) utiliza el término "Capital Psíquico" (p.62-66) compuesto por una serie de variables cognitivas, conductuales y emocionales que a continuación se detallan:

\section{Capacidades cognitivas para adquirir y usar formas de conocimiento:}

\section{Creatividad}

Curiosidad

Motivación para aprender

Sabiduría.

Con estas cuatro variables, cognitivas, podemos decir, que se iniciaría el proceso de la de la metodología Aprendizaje -Servicio.

Se necesita creatividad, para ver dónde encajarán las competencias académicas en un sector poblacional concreto. La curiosidad es necesaria porque nos abre la puerta del descubrimiento, dando paso a la motivación para aprender y conseguir la sabiduría que nos hará discernir lo qué es real y adecuado en el desarrollo de las competencias. El hecho de conseguir desarrollarlas favorece de manera intrínseca y extrínseca el aprendizaje resiliente.

Capacidades emocionales para desarrollar proyectos, superando obstáculos:

Honestidad

Autoestima

Persistencia 
Resiliencia

Las variables emocionales enunciadas están encaminadas a mejorar el perfil psicológico, el cual es susceptible de ser modificado dependiendo de las circunstancias activadoras.

Formar parte de en un proyecto de Aprendizaje-Servicio es garantía de participar en un intercambio emocional que promueve el deseo de ayudar al otro mientras uno es ayudado, recibiendo las cualidades que al efectuar el trabajo se reciben.

\section{Habilidades cívicas para lograr mejor participación ciudadana: \\ Liderazgo \\ Lealtad \\ Compromiso \\ Prudencia \\ El compromiso social que se adquiere al efectuar una labor solidaria, nos empodera para mejo- rar la calidad de vida de las personas o colectivos que van a ser objeto de nuestra ayuda. A su vez nos compromete con el otro, aportando con las dosis de prudencia necesarias para abordar la rea- lidad que el Aprendizaje-Servicio promueve.}

\section{Capacidades para establecer vínculos interpersonales:}

Inteligencia emocional

Amor

Sentido del humor

Empatía y altruismo

Del empoderamiento, la lealtad y el compromiso que se va fomentando en nuestros alumnos, emergerán capacidades para vincularse de una manera empática y altruista.

\section{Sistema de valores como metas que orientan los comportamientos:}

Sentido de justicia

Capacidad para perdonar

Gratitud

Espiritualidad (Casullo, 2005, p.62-66).

Como podemos observar, la resiliencia está incluida dentro de las capacidades emocionales, pero cualquiera de los factores enunciados contribuye a su emergencia.

\section{APRENDIZAJE-SERVICIO: PROMOTOR DE LA RESILIENCIA}

Hemos visto las características del Aprendizaje-Servicio como una pedagogía que promueve muchos beneficios en distintas direcciones y que, a su vez, permite una gran diversidad de puntos de mira. A este respecto, queremos posicionar nuestra mirada en los aspectos que pueden permitir el desarrollo de la resiliencia, entre ellos, lo que se obtiene de empoderar a nuestros alumnos al realizar proyectos donde ellos son los responsables.

Así mismo, hemos podido constatar que el término empoderamiento se vincula a otros términos como pueden ser, el fortalecimiento, la potenciación, o el apoderamiento, y con ellos, se introduce la noción de poder no sólo como fenómeno social, sino también como variable psicológica.

A su vez, "el empoderamiento no sólo es un concepto operativo, sino que también representa un valor que es la base de la transformación cultural" (Sánchez Vidal 2013, p.288). En este sentido, el enfoque de la resiliencia en programas sociales se manifiesta como proceso de interacción en el que intervienen factores que convergen para facilitar el desarrollo humano y social. Además se puede mostrar como una dimensión innovadora que sirve para acompañar mediante la atención y la escucha (Aguilar, Arjona y Noriega, 2016). 


\section{LA METOdOLOGÍA APRENDIZAJE SERVICIO COMO FACTOR EDUCATIVO DE RESILIENCIA}

La educación que permite el desarrollo de las mismas contribuye, de manera absoluta, a la mejora del clima social necesario para seguir desarrollando las destrezas que formarán parte del aprendizaje significativo. Por esa razón, consideramos que la práctica del aprendizaje servicio es optimizante, ya que implica la educación emocional.

Haciendo referencia a lo anteriormente citado, las competencias emocionales están encaminadas a la adaptación personal. Esto sucede desde el momento en que las mismas influyen en el equilibrio homeostático cuando nos enfrentamos a algún problema que requiere que pongamos en práctica las habilidades necesarias.

En base a los resultados expuestos por algunos autores (Folkman y Lazarus, 1988; Echeburúa, 2005; Reeve, 1992; Limonero et al., 2010, citado en Rubio y Puig 2015 p.149-150), existen dos modos de afrontar una situación que nos plantea un problema, uno el directo y otro el indirecto. En cuanto el método directo, se puede establecer dos tipos de respuesta: centrada en la regulación emocional y centrada en el manejo del problema. Se presenta a continuación un resumen de las mismas.

Las respuestas centradas en la regulación emocional se componen de:

Búsqueda de apoyo social. Consiste en pedir ayuda o consejo, así como apoyo social referido a la red de amistades o personas que tiene el sujeto y de las cuales puede recibir ayuda.

Distanciamiento. Tratar de olvidar el problema, restarle importancia, no se trata de negarlo pero sí buscar desconexión.

Autocontrol. Actuar sin precipitarse, controlar las emociones que se producen a lo largo del proceso de afrontamiento de la adversidad.

Aceptación de la responsabilidad. Reconocer la implicación en el problema y en su mantenimiento.

Reevaluación positiva. Encontrar lo bueno de lo malo, reevaluación emocional más centrada en el problema.

Las respuestas centradas en el manejo del problema están centradas en:

Confrontación. Acciones directas dirigidas hacia la situación, dirigiéndose hacia la fuente de estrés e intentar identificar la emoción del momento sin que domine para intentar transformarla.

Planificación de solución de problemas. Encaminados a anticipar los posibles problemas que puedan surgir, teniendo respuestas anticipadas.

Pensar o ejecutar un plan. Elaborar un programa que garantice a priori, la consecución de los objetivos.

Todas estas variables están encaminadas a enriquecer el repertorio personal de los alumnos. El efecto emocional que provoca tener la oportunidad de vivir con el otro, ofreciéndose a través de un servicio personal, modula tanto las expectativas del que ofrece el servicio como las del que lo recibe.

En educación, los conocimientos, las habilidades sociales y las habilidades emocionales deben estar relacionados para que los mecanismos que derivarán en resiliencia se activen. Se necesita una serie de experiencias que presenten algún tipo de dificultad que actuará de precursora de todos los recursos personales necesarios para resolver la situación.

Con todo ello estamos viendo que cada persona y cada alumno parte con una dotación emocional para enfrentarse a los estudios. El Aprendizaje-Servicio como encuadre desde el que el alumno debe aprender, se encargará de transformar esos esquemas, esas mentalidades y costumbres en un trabajo individual, organizacional, y comunitario.

Las necesidades cognitivas y emocionales que todos tenemos pueden ser fácilmente satisfechas en la mayoría de los casos, pero a veces las mismas pueden llegar a ocasionar cierto trauma. Por 
ello, debemos conocer los mecanismos que activan nuestras cogniciones más débiles. La relación con el otro que nos proporciona el Aprendizaje Servicio, nos provee de un marco de comunicación promotor de la resiliencia, ya que "las fuentes interactivas de la resiliencia tienen que ver precisamente con la forma en como el sujeto se apropia de la realidad a través del habla y que abre la posibilidad al comportamiento saludable o de superación de los eventos" (Saavedra 2008, p.31).

\section{APRENDIZAJE-SERVICIO COMO FACTOR DE RESILIENCIA ACADÉMICA}

Uno de los problemas con los que muchos estudiantes se encuentran es con el abandono académico, muchas veces debido a la disonancia existente al tiempo empleado y los beneficios que obtiene. Otras razones por las que el abandono puede estar acechando es por la falta de autoestima que viene acompañada de ansiedad y depresión.

El Aprendizaje-Servicio provee de innumerables beneficios psicológicos encaminados a proteger aspectos psíquicos que podían estar en peligro.

La familia, el colegio, la universidad y la comunidad forman parte del entorno de las personas. $\mathrm{L}$ detección de factores que ayudan a promover la resiliencia es muy importante.

Veamos dos modelos encaminados a promover la resiliencia (Henderson y Milstein, 2003 en Barranco, 2009): Describieron una estrategia de seis pasos para promover la resiliencia en la escuela, pudiendo ser aplicada en los alumnos y en la escuela en general. Es muy útil tanto para diagnosticar como para elaborar un programa que evite los problemas relacionados su falta.

Figura 1. Modelo para construir la resiliencia

Fuente: (Milstein y Henderson 2003, p.31 citado en Barranco 2009, p.31)

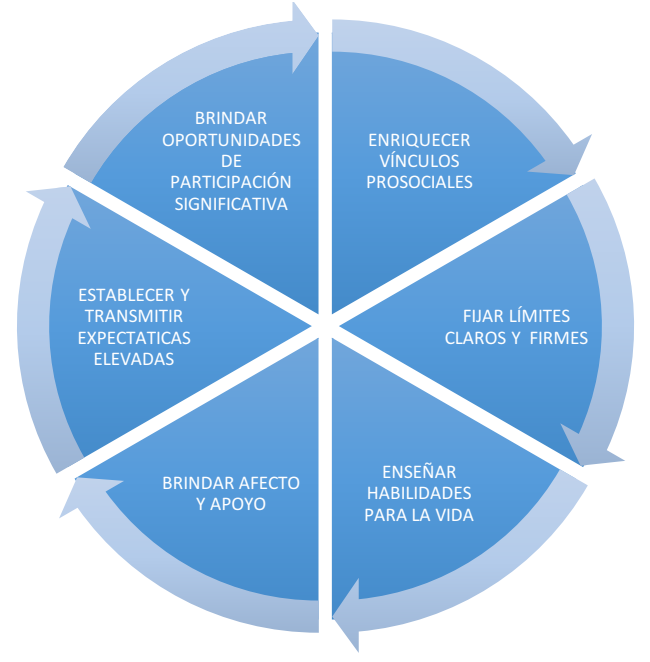

La rueda de la resiliencia está compuesta por seis factores, de los cuales tres tienen un potencial para mitigar los factores de riesgo (brindar afecto y apoyo, establecer y transmitir expectativas elevadas, y brindar oportunidades de participación significativa) otros tres para construir la resiliencia (enriquecer vínculos prosociales, fijar límites, y enseñar habilidades para la vida).

Brindar afecto y apoyo:

Cuando creamos un vínculo de apoyo establecemos una calidad de relación encaminada a detec- 


\section{LA METOdOLOGÍA APRENDIZAJE SERVICIO COMO FACTOR EDUCATIVO DE RESILIENCIA}

tar las necesidades emocionales que deben ser cubiertas, con el fin de resolverlas y transmitir el nivel de confianza óptimo que potencie las fortalezas.

Establecer y transmitir expectativas elevadas:

Cuando nos centramos en las fortalezas, las metas de las que partimos se elevan para encontrar el punto más óptimo posible.

Brindar oportunidades de participación significativa:

Para potenciar las habilidades sociales y académicas, los niños deben colaborar con su grupo de iguales y permitir así un adecuado ajuste en la consecución de sus logros.

Enriquecer vínculos prosociales:

Las relaciones sociales crean una red de apoyo seguro. Éstas a su vez, favorecen el conocimiento recíproco que de ellas emerge, favoreciendo la empatía y estableciendo relaciones de ayuda.

Se refiere a otorgar roles significativos en la consecución de la tarea importantes como solucionar un problema, que hace que las personas se sientan valoradas por sus fortalezas, incrementando su autoestima. Esto ayuda al sujeto a relacionarse con las otras personas y con su alrededor en iguales condiciones.

Fijar límites.

Establecer límites adecuados es una garantía de que las normas establecidas se cumplen favoreciendo el respeto hacia los demás.

Enseñar habilidades para la vida.

Conocimiento compartido entre pares sobre habilidades tales como: cooperación, resolución de conflicto, estrategias de resistencia y asertividad, destrezas comunicacionales, habilidad para resolver problemas y adoptar decisiones y un manejo claro del estrés dependiendo de su edad. (Barranco, 2009, p.31)

Hemos visto como el modelo de Milstein y Henderson proporciona herramientas eficaces para enseñar y aprender cualidades resilientes, pero tenemos que tener en cuenta que la resiliencia es una capacidad que se obtiene si tenemos unas condiciones que la permitan.

Una buena explicación para entender de dónde proviene la resiliencia nos la proporciona la teoría de al apego de Bowlby (1989). Esta teoría defiende la idea de la base segura y hace referencia a un entorno estable que proporciona de manera contingente los cuidados que el bebé necesita, generalmente proporcionado por la madre.

Grotberg, (2013) parte de tres variables personales capaces de fomentar el autoconcepto y por lo tanto la resiliencia. Se inspira en el modelo de Erikson (2002) que define las características de los estadios fundamentales del ciclo vital por el que pasa el ser humano desde la infancia a la vejez y las crisis normativas que del mismo modelo devienen, enfrentándose al reto del estancamiento en cada etapa crítica.

Seguiremos el esquema de Grotberg (2013) para promover la resiliencia:

Yo tengo (hace referencia a los apoyos externos con los que las personas cuentan):

Personas dentro de la familia con las que tengo establecida una base segura y con las que sin duda puedo contar cuando las necesite.

Personas fuera del núcleo familiar que me dan confianza y generan una red de apoyo.

Una conducta de autoestima que garantiza el respeto hacia mí mismo y los demás.

Modelos familiares adecuados que me sirven de referencia a la hora de comportarme.

Una red de apoyo en todos los ámbitos de mi vida, la salud, la educación y la seguridad.

Yo soy (fuerza interior que se desarrolla a través del tiempo y que plantea las siguientes preguntas):

Una persona grata para muchas personas. 
Generalmente tranquilo y con buena disposición.

Alguien que obtiene los planes que se propone a corto medio y largo plazo.

Una persona que se respeta a sí misma y a los demás.

Alguien que siente empatía por los demás y que se preocupa por ellos.

Responsable de mis propias acciones y acepto sus consecuencias.

Seguro de mí mismo, optimista, confiado y tengo muchas esperanzas.

Yo puedo (capacidades interpersonales y de resolución de conflictos):

Generar nuevos modos de hacer las cosas.

Perseverar en una tarea hasta realizarla por dura que sea.

Encontrar el humor en la vida y utilizarlo para reducir tensiones.

Expresar mis pensamientos y sentimientos en comunicación con los demás.

Resolver conflictos en diferentes ámbitos: académico, laboral, personal y social.

Controlar mi comportamiento: mis sentimientos, mis impulsos, demostrar lo que siento.

Pedir ayuda cuando la necesito.

Como podemos comprobar los tres factores, están compuestos por factores externos, fuerza interior y relaciones interpersonales. Estos factores nos permiten incentivar la resiliencia en cualquier etapa, siendo importante saber cuáles de ellos están ya adquiridos con el fin de fortalecerlos.

El punto de partida para activar la resiliencia debe ser una circunstancia que corresponda evolutivamente a nuestro punto de desarrollo y que suponga un desafío a superar. Para ello, será necesario saber detectar las condiciones adversas y posteriormente aplicar cada factor resiliente por separado, utilizándolos de manera interactiva y finalizándola con una evaluación de nuestros resultados en base a si hemos conseguido obtener lo que nos hemos propuesto. (p.20-46)

Tomaremos el modelo yo tengo, yo soy, yo puedo, (Grotberg, 2013) para relacionarlo con la metodología de Aprendizaje-Servicio y ver las condiciones que se activan para favorecer la resiliencia.

Hemos referido al inicio del capítulo que la práctica de la metodología Aprendizaje-Servicio proporciona a los alumnos beneficios personales, sociales y académicos. Ahora vamos a desglosar estos beneficios en base a las características del modelo de Grotberg como medio para promover la resiliencia en nuestros estudiantes.

El docente que se encarga de aplicar la metodología Aprendizaje-Servicio actúa como tutor de resiliencia, dotando a los alumnos de una figura en quién confiar a la hora de resolver las dudas que van surgiendo en su formación académica. Esto conlleva beneficios personales y académicos al reducir la incertidumbre que otros sistemas educativos ofrecen la relación con su tutor.

De la interacción con el ambiente surgirán nuevas ideas para resolver los obstáculos que emerjan de la interacción, permitiendo que los recursos emocionales existentes aumenten y se pongan en marcha las capacidades personales para revisar verificar los resultados desde el principio al final, mejorando el control interno y sabiéndose ayudado en momentos de necesidad.

Teniendo en cuenta las características que distinguen el aprendizaje servicio, tomemos el modelo de Grotberg y veamos de qué modo se ponen en marcha los factores resilientes al aplicar la metodología.

\section{Yo tengo (apoyo externo):}

Una o más personas fuera de mi familia de origen que me entiende y me apoya, que quiere los mejores resultados tanto académicos como humanos, que me fijan límites y explican reglas para que obtenga los logros necesarios.

Profesores que me ayudan a aprender y que son un buen modelo a imitar. 


\section{LA METODOLOGíA APRENDIZAJE SERVICIO COMO FACTOR EDUCATIVO DE RESILIENCIA}

\section{Yo soy (fuerza interior):}

Una persona que agrada a la mayoría de la gente, dado que el trabajo en grupo me permite interactuar con mi grupo de iguales.

He sido el protagonista junto a mis compañeros de todas y cada una de las fases de mi aprendizaje, responsabilizándome de mis acciones me he sentido más seguro y optimista pensando que cuento con apoyos.

Me respeto a mí mismo y a los demás al tener la oportunidad de ver la realidad de otras personas y grupos sintiendo empatía y preocupación.

Figura 2 Aprendizaje-Servicio promotor de resiliencia

Elaboración propia. Aprendizaje- Servicio: características que promueven la Resiliencia.

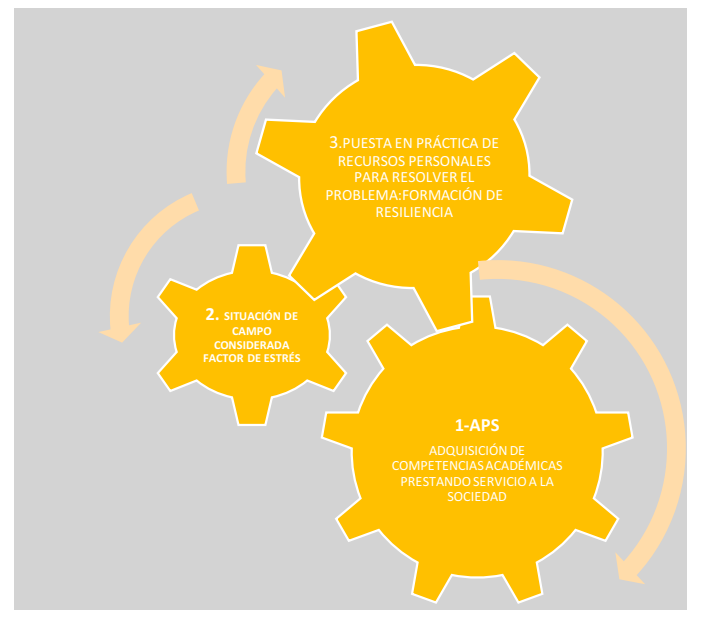

Yo puedo (capacidades interpersonales y de resolución de conflictos):

Generar nuevas ideas 0 nuevos caminos para hacer las cosas, realizar una tarea hasta terminarla.

Encontrar el humor en la vida y utilizarlo para reducir tensiones, expresar mis pensamientos y sentimientos en comunicación con los demás, resolver conflictos en diferentes ámbitos: académico, laboral, personal y social, controlar mi comportamiento: mis sentimientos, mis impulsos, demostrar lo que siento y pedir ayuda cuando la necesito.

Poner mis conocimientos al servicio de la sociedad pongo en marcha todas mis competencias y mi habilidad para pensar y actuar en grupo, detectando las necesidades de los demás.

\section{CARACTERÍSTICAS DEL APRENDIZAJE-SERVICIO COMO TUTOR DE RESILIENCIA}

Un tutor de resiliencia puede tener características muy diversas porque existen muchas y variadas formas de ejercer la tutoría.

Un profesor es un punto de apoyo para cada alumno, y si además le proporciona un medio donde se pueda sentir útil en una situación que le plantea un reto, lo hace más resistente. Pero todo ello tiene que tener un encuadre para que sea eficaz y el encuadre que le proporciona el AprendizajeServicio es la mejor manera de estimular su confianza y favorecer un vínculo incentivante.

El profesor que implementa la metodología Aprendizaje-Servicio se convierte en tutor de resi- 
liencia, esto es, en un mentor empático capaz de imprimir en los alumnos las cualidades necesarias para resolver sus limitaciones y entender que forma parte de una red comunitaria preocupada por el bien común.

Tabla 3

Características de los tutores de Resiliencia

\section{CARACTERÍSTICAS DE LOS TUTORES DE RESILIENCIA \\ LOS TUTORES DE RESILIENCIA PROMUEVEN.}

\begin{abstract}
Visión resiliente
Capacidad de hacer vínculos

Auto- conocimiento de sus fortalezas
\end{abstract}

\author{
Espacios de confianza y seguridad \\ Clima favorable para aprendizajes \\ significativos \\ Espacio mágico de transformación \\ Recursos que dinamizan la \\ autorrealización \\ Recursos que dinamizan la \\ autorrealización
}

Fuente Rubio y Puig (2015, p.157)

El Aprendizaje-Servicio es promotor de todas y cada una de las variables que se incluyen como necesarias, para la formación de la resiliencia. Además, participa de manera circular mediando la relación entre entidades académicas, alumnos y realidad social.

El beneficio que se obtiene aplicando esta pedagogía va mucho más allá de las expectativas docentes y realiza un servicio tutelar que recubre todos a todos y a cada uno de los miembros que en ella participan.

\section{REFERENCIAS BIBLIOGRÁFICAS}

Aguilar, G. N., Arjona, B. A., y Noriega, G. A. (2016). La resiliencia en la educación, la escuela y la vida. Perspectivas Docentes, p. 58.

Banda Castro, A. L., y Morales Zamorano, M. A. (2015). Empoderamiento psicológico: un modelo sistémico con componentes individuales y comunitarios. Revista de Psicología (PUCP), 33(1), 3-20.

Barranco, C. (2009) Trabajo social, calidad de vida y estrategias resilientes. Portularia, vol.I X Núm.2, pp. 133-145.

Bergoglio, J.M (2013) Educar: Exigencia y Pasión. Desafíos para educadores cristianos. Buenos Aires, Argentina: Publicaciones Claretianas.

Bermejo, R. C. (2010). Resiliencia. Una visión positiva para la prevención e intervención desde los servicios sociales. Nómadas. Revista Crítica de Ciencias Sociales y Jurídicas, 27(3), pp.91-103.

Bowlby,J. (1988). Una base Segura: Aplicaciones clínicas de la teoría del apego. Buenos Aires, Argentina: Paidós.

Casullo, M. (2005). El Capital psíquico. Aportes de la Psicología Positiva. Psicodebate 6. Psicología. Cultura y sociedad.

Chiva, Ò., Gómez, J. G., Martínez, R. C., y Peris, C. C. (2016). El aprendizaje servicio como propuesta metodológica para una pedagogía crítica. RIDAS. Revista Iberoamericana de Aprendizaje Servicio, (2), pp.70-94.

Domínguez, B. M. (2014). Aprendizaje-Servicioy Educación Inclusiva. Educación y futuro: Revista de 


\section{LA METODOLOGíA APRENDIZAJE SERVICIO COMO FACTOR EDUCATIVO DE RESILIENCIA}

investigación aplicada y experiencias educativas, (30),pp- 183-208.

Edel, Navarro, R. (2003). El rendimiento académico: concepto, investigación y desarrollo. REICE: Revista Electrónica Iberoamericana sobre Calidad, Eficacia y Cambio en Educación.

Erikson, E. H,(2000). El ciclo vital completado. Barcelona, España: Paidós

Gil, M. I. S., y Garzarán, R. M. R. (2015). Aproximación a los impactos y beneficios del aprendizaje servicio en la Universidad de Zaragoza. RIDAS. Revista Iberoamericana de Aprendizaje Servicio, (1), 9-27.

Guajardo, E. S., Ríos, A. D. C., y Castro, P. S. (2012). Autopercepción de los jóvenes universitarios y resiliencia: construcción de sus particularidades. Katharsis, (14), 89-106.

Grotberg, H.E.( 2013). La resiliencia en el mundo de hoy. Cómo superar las adversidades. Barcelona, España: Gedisa

Manciaux, M. (comp) (2003). La resiliencia: resistir y rehacerse. Barcelona, España : Gedisa.

Musitu, G., \& Buelga, S. (2004). Desarrollo comunitario y potenciación (empowerment). Introducción a la psicología comunitaria, pp. 167-193.

Orteu, M. (2012). Escuelas Resilientes. Recuperado Enero, 7(2014), 2011-12.

Rubio, J. L. y Puig, G.(2015). Tutores de resiliencia: Dame un punto de apoyo y moveré MI mundo. Barcelona, España: Gedisa.

Saavedra Guajardo, E., y Villalta Paucar, M. (2008). Medición de las características resilientes: un estudio comparativo en personas entre 15 y 65 años. Liberabit, 14(14), 32-40.

Tapia, M. N. (2006). Aprendizaje y servicio solidario: algunos conceptos básicos. Documento recuperado el, 3(06). 